\title{
Automatic Leveling System of Precision and Heavy Load Platform
}

\author{
Zhenting Liu \\ School of Mechanical Engineering and Automation \\ BeiHang University \\ Beijing, China \\ ztliu@buaa.edu.cn
}

\author{
Dazhai Li \\ School of Mechanical Engineering and Automation \\ BeiHang University \\ Beijing, China \\ lidazhai@buaa.edu.cn
}

\begin{abstract}
This paper mainly introduces a set of automatic leveling system for high precision and heavy load platform and describes the principle of system operating, the component of hardware and software. The angle sensor is the basis of leveling, and the electric push rod is the guarantee of leveling. Motion control card controls the movement of the electric push rod, so that the platform inclination angle changes, according to the feedback of the angle sensor to determine the platform is level or not. The system can automatically complete the automatic leveling of the platform, has a friendly operation interface with parameter setting and state display. It also has limited protection and overload monitoring which is safe and reliable. The position accuracy of the electric push rod can be ensured by using the electric push rod as the driving device of the servo motor and the position feedback by the external Grating ruler. Therefore, it can meet the high accuracy requirements under heavy load conditions.
\end{abstract}

Keywords- leveling; high precision; heavy load; electric push rod; angle sensor

\section{INTRODUCTION}

Leveling device has a very wide range of use in the military, engineering, and other fields, such as the military radar, launch vehicles, large engineering vehicles and some platform equipment, etc. The common automatic leveling method concludes the hydraulic type automatic leveling and the electromechanical type automatic leveling. Hydraulic automatic leveling can meet the needs of large load, but the hydraulic system is more complex, also the control is difficult and not easy to maintain. For electromechanical type automatic leveling, it is relatively easy to control and easy to maintain. What is more, it is of high accuracy and the load is moderate. Considering the leveling accuracy of the platform has a great impact on the accuracy of the equipment, this system adopts the 4 point support platform, which is used for the automatic adjustment of the mechanical and electronic level.

\section{AUTOMATIC LEVELING SYSTEM MODEL}

The model of the automatic leveling of the four support platform is shown in Fig. 1, which includes 4 sets of supporting legs, a two axis electronic angle sensor and a control box. The horizontal coordinates are XOY, and the 4 legs are N1, N2, N3, N4, respectively. N1, N2 is a fixed electric push rod and N3, N4 is the electric push rod that can be tilted. In order to guarantee the high precision requirement, the lower end of the fixed rod is fixed on the base, and the upper end is connected with the platform through the planar hinge; the upper and lower ends of the electric push rod which can be tilted connected with the base and the platform by the planar hinge respectively. Therefore, the platform must ensure that the level of $\mathrm{X}$ direction in the early time of installation, in the latter part of the use of the process should always ensure that the level of $\mathrm{X}$ direction. The tilt sensor is digital dual axis obliquity sensor and the control box contains industrial control computer, motion control card, data acquisition card, protective switch, relay, etc.

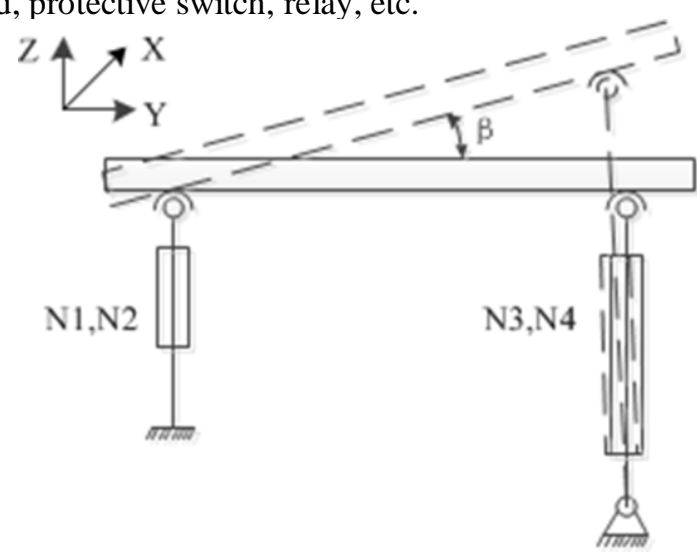

Figure 1. System model

\section{AUTOMATIC LEVELING SYSTEM STRUCTURES}

\section{A. leveling method}

As a benchmark platform, it is not required for its mobility, and it does not need to consider the complex ground environment. The mechanical structure of the platform does not allow the $\mathrm{X}$ direction to have a tilt, so as to improve the accuracy of the $\mathrm{X}$ direction, and then the platform is mainly to adjust the $\mathrm{Y}$ direction. And the leveling process must ensure that the position of the servo motor is synchronized. For this rigid platform, the speed and position of the electric push rod are not the same as when using multi point adjustment. The connection mode of the electric push rod is connected with the plane hinge, and the $\mathrm{X}$ direction is required in the installation and use, otherwise it will cause the electric push rod stuck, the servo motor overload fault, more serious damage to the device and the occurrence of unexpected danger. Therefore, the platform is not suitable for the adjustment of multi - 
point adjustment, the only way can be used is unidirectional adjustment.

The unidirectional adjustment of the coordination is good and the electric push rod only rise but does not fall when leveling so as to reduce the error of the backlash of the servo drive. When $\beta>0, \mathrm{~N} 1, \mathrm{~N} 2$ sync up to lift; when $\beta$ $<0, \mathrm{~N} 3, \mathrm{~N} 4$ sync up to lift.

\section{B. System operation principle}

By receiving the upper computer interface operation command, the platform turns in the adjustment mode. The industrial control computer sends the position control movement instruction to the motion control card. The movement control card analyzes the instruction of the industrial control computer and sends the pulse and instruction to the drive servo driver, controlling the electric push rod to extend out. Through the motion control card's synchronous motion control function, realizes the electric push rod synchronous upward to the top, causes the platform and the fixed support to separate.

After the platform and the fixed support is completely separated, the dual axis obliquity sensor begins to detect the angle between two intersecting lines( $\mathrm{X}, \mathrm{Y}$ axis) and the base plane, then the angle can be transmitted to the industrial control computer through the 485 serial port in real time. The industrial computer compares the values that collected from sensor with the error allowable values. When the error value exceeds the limit, the industrial control computer generates and sends control commands through the motion control card, and controls the speed
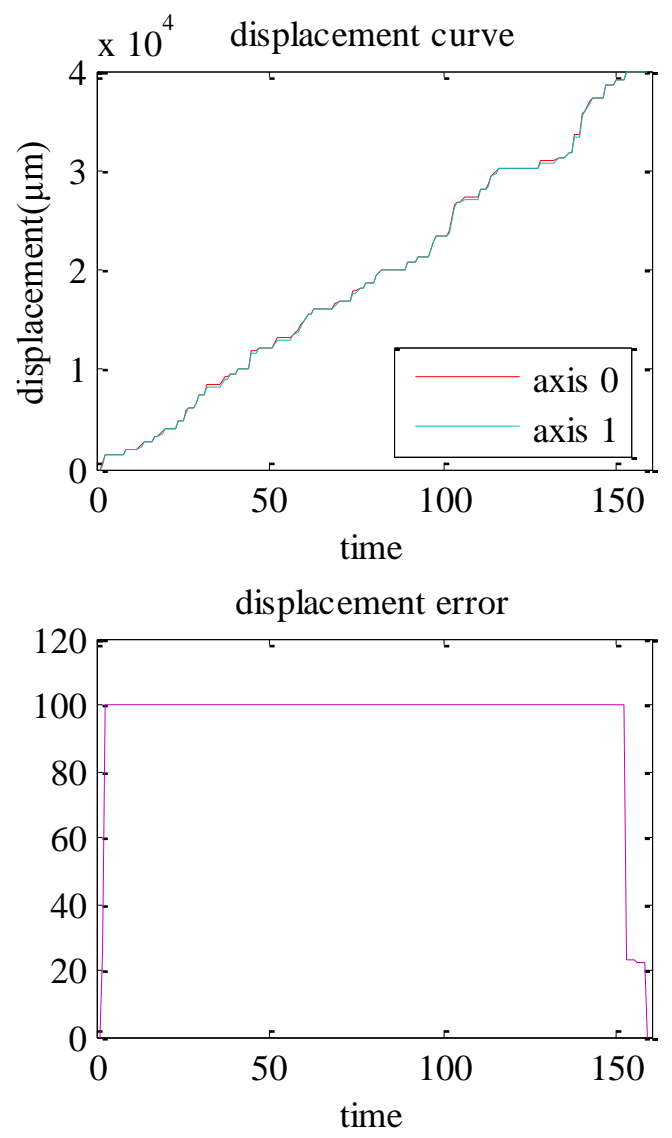

and position of the electric push rod to ensure the error is in the allowable range.

At the progress of levelling, monitor the weighing sensors' reading which installed on each electric push rod in real-time and then sent the thrust force of each electric push rod back to the industrial computer through the data acquisition card $\mathrm{AD}$ conversion function. At the same time, the pulse count of the grating ruler that is connected to the electric push rod is read in real time, ensuring that each electric push rod displacement synchronization. Within the allowable error range, the thrust force of the electric push rod is compared with the pre load carrying capacity by the industrial computer to ensure that the virtual leg will not appear. According to the relevant algorithm, once the virtual leg is in the trend, the industrial computer compensated for the displacement, in order to achieve automatic error compensation.

The limit switch signal of electric push rod, bearing capacity and the reading of digital dual axis angle sensor are displayed on the interface.

\section{Key factors of leveling}

The accuracy and stability of the digital dual axis angle sensor has a direct impact on leveling accuracy of the platform. The high precision synchronous motion of the electric push rod is the key to the leveling time and precision of the system.

Therefore, it is very important to choose the high precision and high speed digital angle sensor. The angle sensor used in this paper is QT-620T. It is a high precision angle measuring product which is developed by the
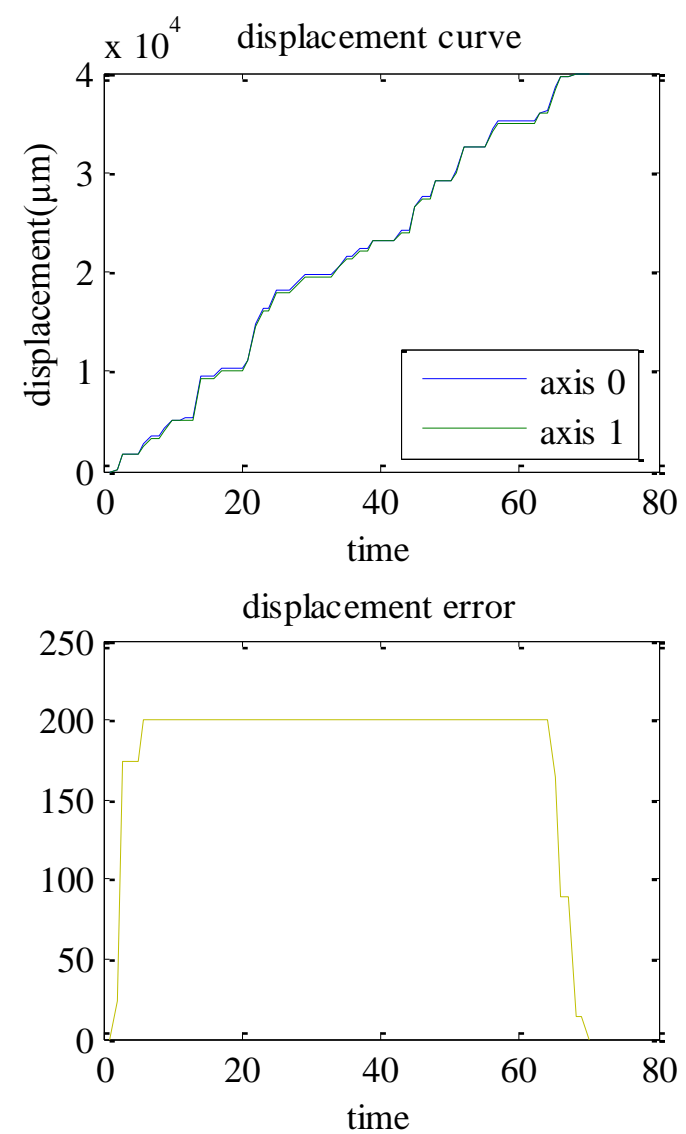

Figure 2. Low speed synchronization and high speed synchronization 
application of industrial control, engineering measurement, measurement and detection, and the system is built with high precision 24 bit A/D differential converter, equipped with powerful digital processing system. The seismic performance is good, the detection range from -30 degrees to +30 degrees, and the detection accuracy range from 0.02 to 0.02 degrees. The digital angle sensor has a data update rate of 10 times per second, and the transmission rate of default $9600 \mathrm{bps}$. By using the standard MOSBUS industrial protocol, the digital angle sensor connects with the industrial computer through a 485 serial port.

At the time of leveling, the digital angle sensor detects the inclination angle of platform in real time, and then sent the angle date to the industrial control computer. Industrial control computer receives the data and transforms it into angle value. If the angle data collected to meet the expected requirements then stop testing, and stop the operation of the electric push rod. If the angle data is not met the expected demand, then the industrial control computer will determine the high point of $\mathrm{Y}$ axis based on the angle data, and then synchronize the lower side of the electric push rod, until the angle to meet the expected requirements. In the whole process, the $\mathrm{X}$ direction is always kept within the limit error, which requires that the precision of the synchronous motion must be within the limits.

Electric push rod is driven by servo motor. The model of the servo motor is Panasonic MDME152GCH, and the driver model is MDDKT5540CA1. The reduction ratio of the electric push rod is 30 , the helical pitch is $10 \mathrm{~mm}$, the positioning accuracy is $0.01 \mathrm{~mm}$, the rated thrust is 76000 $\mathrm{N}$, and the external grating ruler's resolution is $0.005 \mathrm{~mm}$. Set the servo motor to rotate one circle every 10000 pulses. Then sent 300000 pulses to the motor servo, the electric push rod will move a distance of $10 \mathrm{~mm}$. Motion control card is IMC3162E, with a variety of motion control functions. The motion control card sends pulse and direction to the motor servo driver and control the lift of the electric push rod driven by the servo motor.

In this paper, the motion control card's electronic gear mode is used to realize the synchronous movement of electric push rod. The electronic gear mode is that the driven shaft follows the motion of driving shaft in accordance with the set ratio. In order to use this function, you only need to configure the transmission ratio, set the driving shaft number, set the driven shaft to follow the main encoder speed. After the setup is finished, the synchronization can be realized simultaneously with the driving shaft and the driven shaft. Since the displacement of the electric push rod is consistent, the driving ratio of the driving shaft and the driven shaft is set to 1. After each synchronized movement is completed and stopped, the cancellation of synchronization can be made.

It shows the displacement curve and the error curve of the drive shaft and the driven shaft at different speeds in Fig .2. From the experimental results it seems that the synchronization error and the speed of synchronization are positively related.

\section{Hardware structure}

The hardware structure diagram is shown in Fig .3. Because of this automatic leveling platform equipment is mainly used for large and heavy parts of the measurement, so its accuracy, stability, reliability requirements. At the same time, the data should be collected and processed in real time. Therefore, the system chooses the industrial control computer as the core, supplemented by the motion control card, data acquisition card, digital dual axis angle sensor, grating ruler and other peripheral equipment.

Controller part: Industrial computer is chosen as the core of the control, which can be friendly and compatible with the data acquisition card and other peripherals, which has good stability in order to adapt to the harsh industrial environment. It can also achieve real-time data acquisition and processing.

Communication part: Including the communication between industrial control computer with the motion control card and the digital dual axis angle sensor.

The industrial control computer is connected with the digital dual axis angle sensor through the 485 serial port. Through the upper computer software, the angle data is directly read and calculated by analyzing the data frame. The industrial control computer and motion control card are connected with the real time Ethernet, and the optimized control protocol is implemented to realize high speed and high reliability.

Sensor part: The system platform needs to detect the level of the platform and the state of the force and position of the electric push rod.

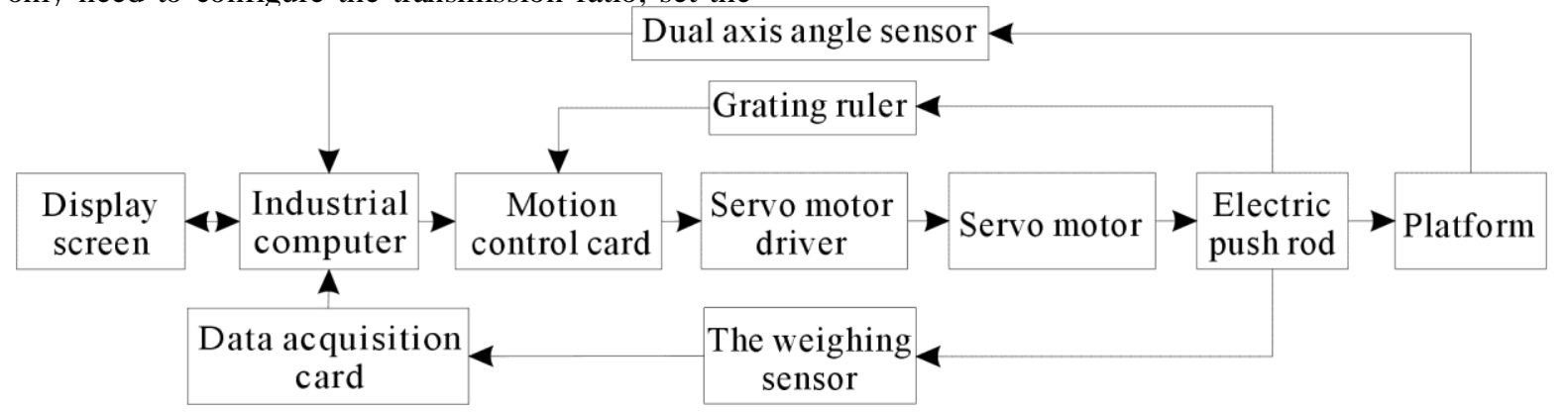

Figure 3. System hardware structure

The level of the platform depends on the digital dual axis angle sensor. Its performance and reliability directly affect the accuracy and reliability of the platform. The thrust sensor of the electric push rod can detect the force of the load of the electric push rod in real time, avoid the appearance of the virtual leg and the system's electric push rod jammed.

Implementation part: By accepting the instructions from the motion control card, the position control of the full closed loop is realized. The actuator is an electric push rod, driven by a servo motor. The servo driver and the 
servo motor constitute the inner loop, the grating ruler is used as outer ring. They together constitute the full closed loop position control. It can meet the speed, acceleration and position control of the electric push rod, and realize the function of the rise, fall and maintain.

\section{E. Software structure}

Leveling device is a platform with four supports. Before the start of the leveling, each electric push rod synchronized elevation of a certain distance, making the platform and the fixed support separation, this process requires $\mathrm{N} 1, \mathrm{~N} 2$ synchronization and N3, N4 synchronization. At the beginning of leveling, the digital dual axis angle sensor detects the higher side of the $\mathrm{Y}$ direction. And then synchronize the two electric push rods at the lower side of the $\mathrm{Y}$ direction until the angle meets the requirements. In the process of leveling, each electric push rod only rise not fall.

The main program flow of the automatic leveling system is shown in Fig .4.

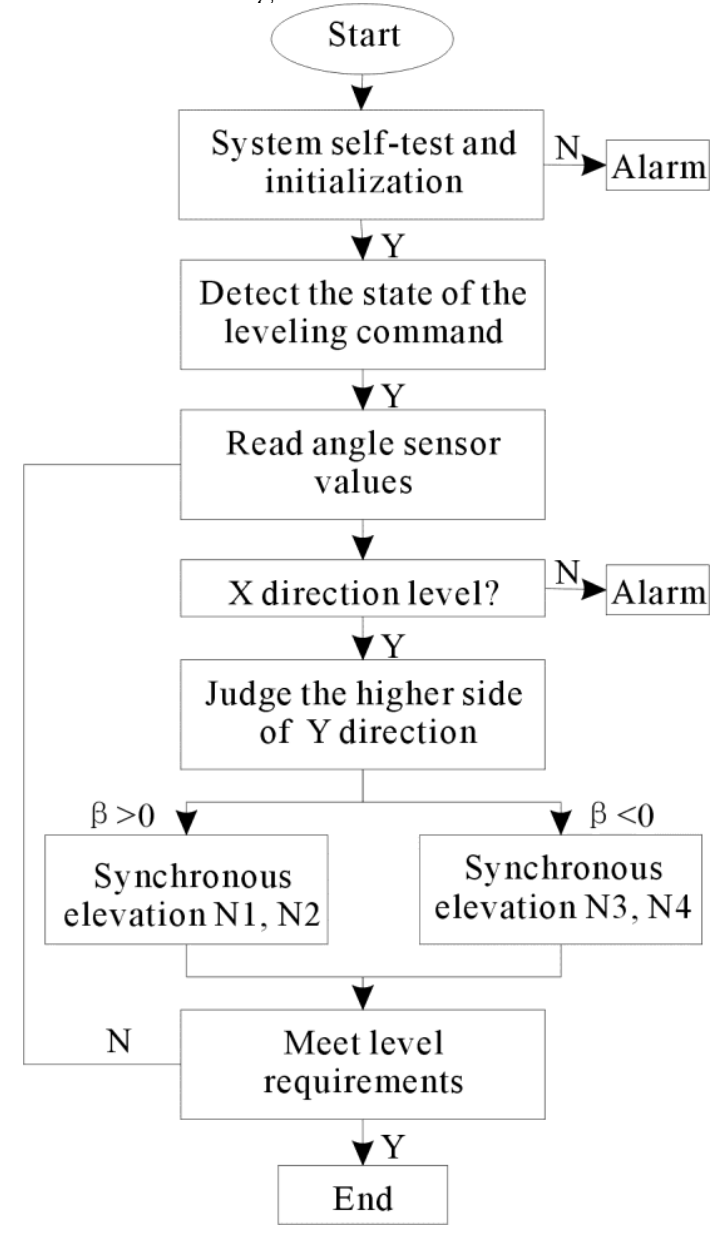

Figure 4. System main program flow

Virtual leg is a common problem of the four fulcrum platform. The system strictly control the real-time position of the electric push rod, and the bearing capacity of the electric push rod as well as the angle of the angle sensor to monitor the real-time monitoring, to avoid a certain electric push rod or the $\mathrm{X}$ direction of the tilt angle beyond the allowable range. This will ensure that the normal operation of the equipment platform, effectively avoid failure and danger.
The key lies in the synchronous movement of the electric push rod. The synchronous motion depends on the position control of the servo motor and the position feedback of the external grating. In each synchronized motion of the leveling process, the target distance is guaranteed to be the same and the position in the process of synchronization is also the same. So the feedback pulse of the grating ruler is an important guarantee for the synchronous motion. The drive of the electric push rod is the Panasonic servo motor, and the motion control card IMC has the electronic gear to realize the multi axis linear synchronization. IMC electronic gear ratio is 48 , the high 16 is an integer part, the low 32 is the fractional part, can achieve high precision gear drive.

\section{CONCLUSION}

In this paper, the control system of high precision and heavy load platform based on the four point support is studied. The servo motor driven electric push rod has the function of synchronous motion, and the accuracy of synchronization is different at different speeds. At low speed, it can achieve better accuracy, and the synchronization precision is approximately linear with the speed of synchronous motion. At present, the system has already carried on the preliminary debugging. Based on the synchronous motion of the electric push rod and the electronic axis angle sensor, the leveling accuracy of the platform can be stabilized between $-0.02^{\circ}$ and $+0.02^{\circ}$. If you choose the higher accuracy of the tilt sensor or reduce the speed of synchronous operation of the electric push rod, then the platform will be more high accuracy.

\section{REFERENCE}

[1] Yu-xia L I, Da-zhai L I. Automatic Leveling System based on AD Conversion[J]. ICCAS 2011, 2011: 411-414.

[2] Jean-Paul Louis.Control of Synchronous Motors[M].2013.

[3] Feng Yi, Chen Bojin. Control system of automatically leveling of vehical-borne radars[J]. Journal of Huazhong University of Science and Technology: Natural Science Edition, 2004, 32(6): 66-68.

[4] Wen Bangchun ... [et al.].. Vibratory synchronization and controlled synchronization in engineering[M].Science Press.2009.

[5] SUN J, WU Y, CHEN S, et al. Intelligent leveling system for suspended access platforms[J]. Chinese Journal of Construction Machinery, 2011, 2: 010.

[6] Guangwei L, Xinfang X. Electromechanical Automatic Leveling System[J]. Ordnance Industry Automation, 2013, 32(3): 1-2.

[7] Xiao Y, Pang Y, Ge X, et al. Synchronous control for highaccuracy biaxial motion systems[J]. Journal of Control Theory and Applications, 2013, 11(2): 294-298.

[8] Chauhan V P P V, Patel V P. Multi-motor Synchronization Techniques[J]. International Journal of Science, Engineering and Technology Research (IJSETR), 2014, 3: 319-322.

[9] Fang S, Liu Y, Otsubo H, et al. An automatic leveling method for the stage of precision machining center[J]. The International Journal of Advanced Manufacturing Technology, 2012, 61(1-4): 303-309.

[10] Tian C P, Tang G Y, Su H, et al. Position prediction and delay compensation on leveling systems[C]//Control Conference (CCC), 2014 33rd Chinese. IEEE, 2014: 7767-7771.

[11] Yau H T. Chaos synchronization of two uncertain chaotic nonlinear gyros using fuzzy sliding mode control[J]. Mechanical Systems and Signal Processing, 2008, 22(2): 408-418..

[12] Yao-Hui W, Jian-Rong S, Ke-Jin B. An Automatic Level Adjustment Device Based on STM32[C]//Intelligent Computation Technology and Automation (ICICTA), 2014 7th International Conference on. IEEE, 2014: 56-59. 